Gueriau, P., et al., 2020, Oxidative conditions can lead to exceptional preservation through

phosphatization: Geology, v. 48, https://doi.org/10.1130/G45924.1

\title{
Oxidative conditions can lead to exceptional preservation
}

\section{through phosphatization}

\section{Pierre Gueriau ${ }^{1,2,3}$, Sylvain Bernard ${ }^{4}$, François Farges $^{4}$, Cristian Mocuta ${ }^{1}$, Didier B.}

\section{Dutheil $^{5}$, Thierry Adatte ${ }^{3}$, Brahimsamba Bomou ${ }^{3}$, Marie Godet ${ }^{2}$, Dominique}

Thiaudière $^{1}$, Sylvain Charbonnier ${ }^{5}$, and Loïc Bertrand ${ }^{2,1,6}$

${ }^{1}$ Synchrotron SOLEIL, l'Orme des Merisiers, BP 48 St Aubin, 91192 Gif-sur-Yvette, France

${ }^{2}$ Université Paris-Saclay, CNRS, ministère de la Culture, UVSQ, MNHN, Institut photonique

d'analyse non-destructive européen des matériaux anciens, 91192, Saint-Aubin, France

${ }^{3}$ Institute of Earth Sciences, University of Lausanne, Géopolis, CH-1015 Lausanne,

Switzerland

${ }^{4}$ Muséum National d'Histoire Naturelle, Sorbonne Université, CNRS, UMR 7590, Institut de

Minéralogie, Physique des Matériaux et Cosmochimie, Paris, France

${ }^{5}$ Muséum national d'Histoire naturelle, Sorbonne Université, CNRS UMR 7207, CR2P,

Centre de Recherche en Paléontologie - Paris, 8 rue Buffon, 75005, Paris, France

${ }^{6}$ Université Paris-Saclay, ENS Paris-Saclay, CNRS, Photophysique et Photochimie

Supramoléculaires et Macromoléculaires, 91190, Gif-sur-Yvette, France 


\section{METHODS}

\section{Synchrotron X-ray fluorescence major-to-trace elemental mapping}

Micro X-ray fluorescence ( $\mu$ XRF) maps were collected at the DiffAbs beamline of the SOLEIL synchrotron (Gif-sur-Yvette, France), using an excitation energy of $17200 \mathrm{eV}$ selected for excitation of K-lines form elements up to yttrium and L-lines up to uranium. Note, however, that as the experiment was performed in air low energy XRF photons (produced by light elements such as phosphorus or low energy L- and M-lines) are absorbed by air molecules before reaching the detector, therefore narrowing the detection to elements from chlorine to uranium. The incoming X-ray beam was collimated by 2 bendable mirrors, monochromatized using a $\mathrm{Si}(111)$ double-crystal monochromator (energy resolution: $\Delta \mathrm{E} / \mathrm{E} \approx$ $10^{-4}$ ) and focused using Kirkpatrick-Baez (KB) mirrors (Kirkpatrick and Baez, 1948) down to a spot size of $11 \times 7 \mu \mathrm{m}^{2}(\mathrm{H} \times \mathrm{V}$, full width at half maximum, fwhm). The sample was mounted on a xyz scanner stage allowing $\pm 12 \mathrm{~mm}$ movements with micrometer accuracy. The sample was oriented at $45^{\circ}$ to the incident beam and at $45^{\circ}$ to the 4 -element silicon drift XRF detector (SDD, Vortex ME4, Hitachi High-Technologies Science America, Inc., total active area: 170 $\mathrm{mm}^{2}$ ) placed in the horizontal plane.

All elemental distributions presented herein have been reconstructed from full spectral decomposition performed with the PyMCA data-analysis software (Solé et al., 2007) using a batch-fitting procedure, polynomial baseline subtraction, and assuming a Pseudo-Voigt peak shape. Fitting and experimental parameters, in particular the photon flux in the focused spot, were determined from a fit on the $\mu \mathrm{XRF}$ spectrum collected on a fluorapatite standard (AS1045-AB, SPI Supplies). Even though full quantification of trace elements using XRF is hampered by the local heterogeneity of the tissues that limits the precision of corrections for matrix X-ray re-absorption, statistics were sufficient to estimate the semi-quantitative 
contents of the elements. In particular, comparison of contents in REEs is made easier by the comparable information depths.

\section{Synchrotron X-ray absorption spectroscopy}

\section{Micro X-ray absorption spectroscopy at the Ce $\mathrm{L}_{3}$-edge}

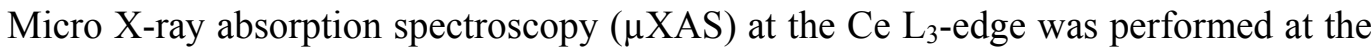
LUCIA beamline of the SOLEIL synchrotron. The X-ray beam was monochromatized using a $\mathrm{Si}(111)$ double-crystal monochromator and focused using $\mathrm{KB}$ mirrors downstream to a diameter of $3 \mu \mathrm{m}$ (fwhm). Energy calibration was performed by setting the first edge inflection point of data from an iron foil at $7112 \mathrm{eV}$. Loose samples from the fossils were mounted on a small copper slab that can hold samples up to $\sim 24 \times 18 \mathrm{~mm}^{2}$-large, placed on a micrometric xyz scanner stage under primary vacuum. The sample was orientated at $85^{\circ}$ to the incident beam and at $5^{\circ}$ to a 4-element SDD XRF detector in the horizontal plane (QUAD SD XFlash Detector 5040, Bruker AXS; total active area, $40 \mathrm{~mm}^{2}$ ), so as to maximize spatial

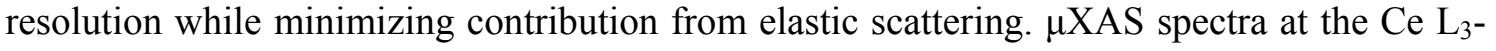
edge were collected at room temperature in fluorescence mode in the 5680-5860 eV range. Energy step sizes were $2 \mathrm{eV}$ between 5680 and $5710 \mathrm{eV}, 0.3 \mathrm{eV}$ between 5710.3 and 5746 $\mathrm{eV}, 1 \mathrm{eV}$ between 5747 and $5790 \mathrm{eV}$, and $2 \mathrm{eV}$ between 5792 and $5860 \mathrm{eV}$. A $1 \mathrm{~s}$ counting time was used per energy step and 2 spectra were cumulated.

Spectra were processed through self-written routines using the $\mathrm{R}$ statistical environment ( $\mathrm{R}$ Development Core Team, 2011) by subtracting the pre-edge and the absorption background. In order to assess the contributions of $\mathrm{Ce}(\mathrm{III})$ and $\mathrm{Ce}(\mathrm{IV})$ species and thus to determine the $[\mathrm{Ce}(\mathrm{IV})] /[\mathrm{Ce}($ tot $)]$ ratio, the spectra were decomposed in a linear combination of spectra from $\mathrm{Ce}(\mathrm{III})\left(\mathrm{NO}_{3}\right) 3 \cdot 6 \mathrm{H}_{2} \mathrm{O}$ and $\mathrm{Ce}(\mathrm{IV})\left(\mathrm{SO}_{4}\right)_{2}$ standards previously measured at the LUCIA beamline by Janots et al. (2015). 


\section{Micro X-ray absorption spectroscopy at the Fe K-edge}

$\mu \mathrm{XAS}$ at the Fe K-edge was performed at the DiffAbs beamline of the SOLEIL synchrotron in the 7034-7400 eV range with a 1 eV energy step in the XANES region (7095$7175 \mathrm{eV})$, using the same geometry as in $\mu \mathrm{XRF}$, and a beam size of $9 \times 7 \mu \mathrm{m}^{2}(\mathrm{H} \times \mathrm{V}$ fwhm). The counting time was set to $2 \mathrm{~s}$ per energy step. Energy calibration was performed by setting the first edge inflection point of data from an iron reference foil at $7112 \mathrm{eV}$.

Spectra were normalized using the XAFS data analysis program (Winterer, 1997) and corrected from self absorption using the FLUO package by D. Haskell (www.aps.anl.gov/ haskel/FLUO). As fluorescence mode is used to collect the XAFS spectra, self absorption was easily detected through characteristic spectral distortions in the

edge region (e.g. severe flattening at the edge crest). Re-absorption of the emitted x-ray beam is due to the presence of Fe-rich phases in the probed volume. Normalized $\mu$ XAFS spectra were decomposed in frequency using continuous Cauchy wavelet transforms (CCWT, see Munoz et al., 2003 for details of the method), a three-dimensional representation of the wave vector $(k)$, the interatomic distance uncorrected for phase-shifts $\left(R^{\prime}\right)$, and the continuous decomposition of the EXAFS amplitude terms (CCWT modulus). Such 3D decomposition brings more constraints to the various atomic pair contributions to the XAFS spectra as compared to simpler XANES spectral comparison methodologies. This allows a more robust identification of the chemical environment of the target atom present in the sample.

\section{Elemental Quantifications}

Inductively coupled plasma - mass spectrometry (ICP-MS) and atomic emission spectroscopy (ICP-AES) quantifications were carried out at Institut des Sciences Analytiques (UMR 5280 CNRS, Lyon, France) and at the Service d'Analyse des Roches et des Minéraux (CRPG, UMR 7358 CNRS-INSU, Vandœuvre-lès-Nancy) using ICP-AES (iCAP, 6500 
DUO) and ICP-MS (Q X7, Thermo Scientific) spectrometers for major elements and trace elements, respectively.

\section{SEM imaging}

Scanning electron microscopy (SEM) imaging and induced X-ray emission spectroscopy was performed at the MNHN microscopy platform in backscattered electron (BSE) mode using a Tescan SEM (VEGA II LSU, low vacuum: 10-48 Pa, $20 \mathrm{kV}$ ), and at the IPANEMA laboratory using a ZEISS FEG-SEM (Supra55VP, 3-5 kV, gold metallization).

\section{X-ray diffraction}

X-ray diffraction (XRD) was performed on powders prepared from a fossil microbial mat and the surrounding sedimentary matrix using a conventional $\mathrm{Cu}-\mathrm{K} \alpha(\lambda=1.5418 \AA)$ powder diffractometer (D2 Phaser, Bruker, Germany) at the SOLEIL synchrotron chemistry laboratory equipped with a Ceramic KFL X-ray tube source and a 1D-linear LynxEye detector, in the following experimental conditions: $30 \mathrm{kV}$ acceleration voltage of the tube; 10 $\mathrm{mA}$ filament current; $2 \theta$ range: $10-70^{\circ} ; 0.02^{\circ}$ angular step, $1 \mathrm{~s}$ counting time. Phase identification was carried out using the Match! software making use of the International Centre for Diffraction Data (ICDD)-PDF 2015 database.

\section{REFERENCES CITED}

Janots, E., Bernier, F., Brunet, F., Munoz, M., Trcera, N., Berger, A., and Lanson, M., 2015, $\mathrm{Ce}(\mathrm{III})$ and $\mathrm{Ce}(\mathrm{IV})$ (re)distribution and fractionation in a laterite profile from Madagascar: Insights from in situ XANES spectroscopy at the Ce $\mathrm{L}_{\mathrm{III}}$-edge: Geochimica et Cosmochimica Acta, v. 153, p.134-148, doi:10.1016/j.gca.2015.01.009. 
Kirkpatrick, P., and Baez, A.V., 1948, Formation of optical images by X-rays: The Journal of the Optical Society of America, v. 38, 766-774, doi:10.1364/JOSA.38.000766.

Munoz, M., Argoul, P., and Farges, F., 2003, Continuous Cauchy wavelet transform analyses of EXAFS spectra: A qualitative approach: American mineralogist, v. 88, p. 694-700, doi:10.2138/am-2003-0423.

R Development Core Team, 2011, R: a language and environment for statistical computing: Vienna, Foundation for Statistical Computing.

Solé, V., Papillon, E., Cotte, M., Walter, P., and Susini, J., 2007, A multiplatform code for the analysis of energy-dispersive X-ray fluorescence spectra: Spectrochimica Acta Part B: Atomic Spectroscopy, v. 62, p. 63-68, doi:10.1016/j.sab.2006.12.002.

Winterer, M., 1997, XAFS-A data analysis program for materials science: Le Journal de Physique IV7, p. C2-243-C2-244, doi:10.1051/jp4/1997182.

\section{INSTITUTIONAL ABBREVIATIONS}

MNHN: Muséum national d'Histoire naturelle, Paris (France); MNHN.F refers to collection numbers from the MNHN Collections.

MHNM: Musée d'Histoire naturelle de Marrakech, Marrakesh (Morocco); MHNM-KK-OT refers to collection numbers for the "Kem-Kem - Oum Tkout" material collected 


\section{FIGURES}
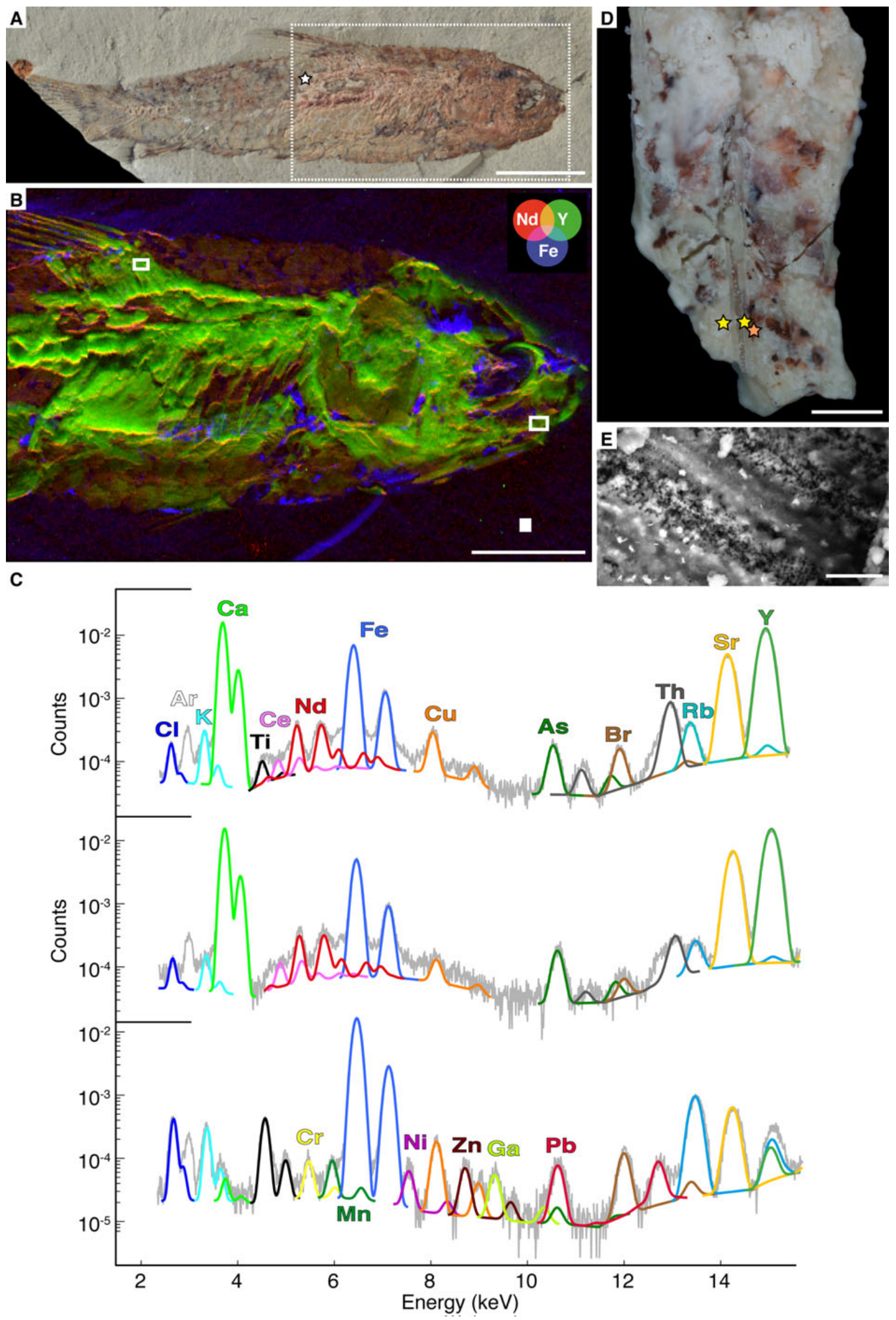
Figure DR1. Synchrotron micro-X-ray fluorescence $(\mu \mathrm{XRF})$ mapping of major-to-trace elements of MHNM-KK-OT 10, and location of XAS and REE patterns data. A: Optical photograph of a teleost fish (MHNM-KK-OT 10). B: False color overlay of neodymium (red), yttrium (green) and iron (blue) distributions from the anterior part of the specimen (corresponding to the dotted white bow area in A). Acquisition parameters: $60 \times 60 \mu \mathrm{m}^{2}$ scan step, 117,820 pixels, $100 \mathrm{~ms}$ counting time per pixel. C: Average XRF spectra and main elemental contributions from the box areas in B. Top and middle spectra are respectively characteristic of the fish jaw (average spectrum from 80 pixels) and dorsal fin muscles (average spectrum from 56 pixels), and correspond to the spectra quantified for reconstructing the partial PAAS-normalized REE patterns presented in Fig. DR5A. Bottom spectrum shows the composition of the clayey matrix (average spectrum from 64 pixels). Note that the unlabeled peak between $\mathrm{Cl}$ and $\mathrm{K}$ is a XRF Ar-peak due to the excitation of Ar in the air (ca. $0.93 \%$ between the sample and the detector. D: Optical photograph of a loose sample of soft-tissues including bone from the MHNM-KK-OT 10 (origin: white star in A). The yellow

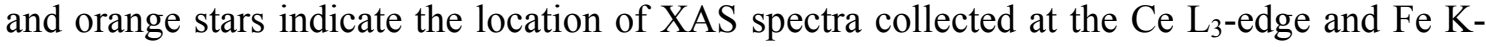
edge, respectively presented in Fig. DR5B and Figs. 3H, 3I. E: SEM image (BSE mode) of the muscle fibers preserved on the sample in D. Scale bars: $A=1 \mathrm{~cm} ; B=5 \mathrm{~mm}$; $=500$ $\mu \mathrm{m} ; \mathrm{E}=10 \mu \mathrm{m}$. 

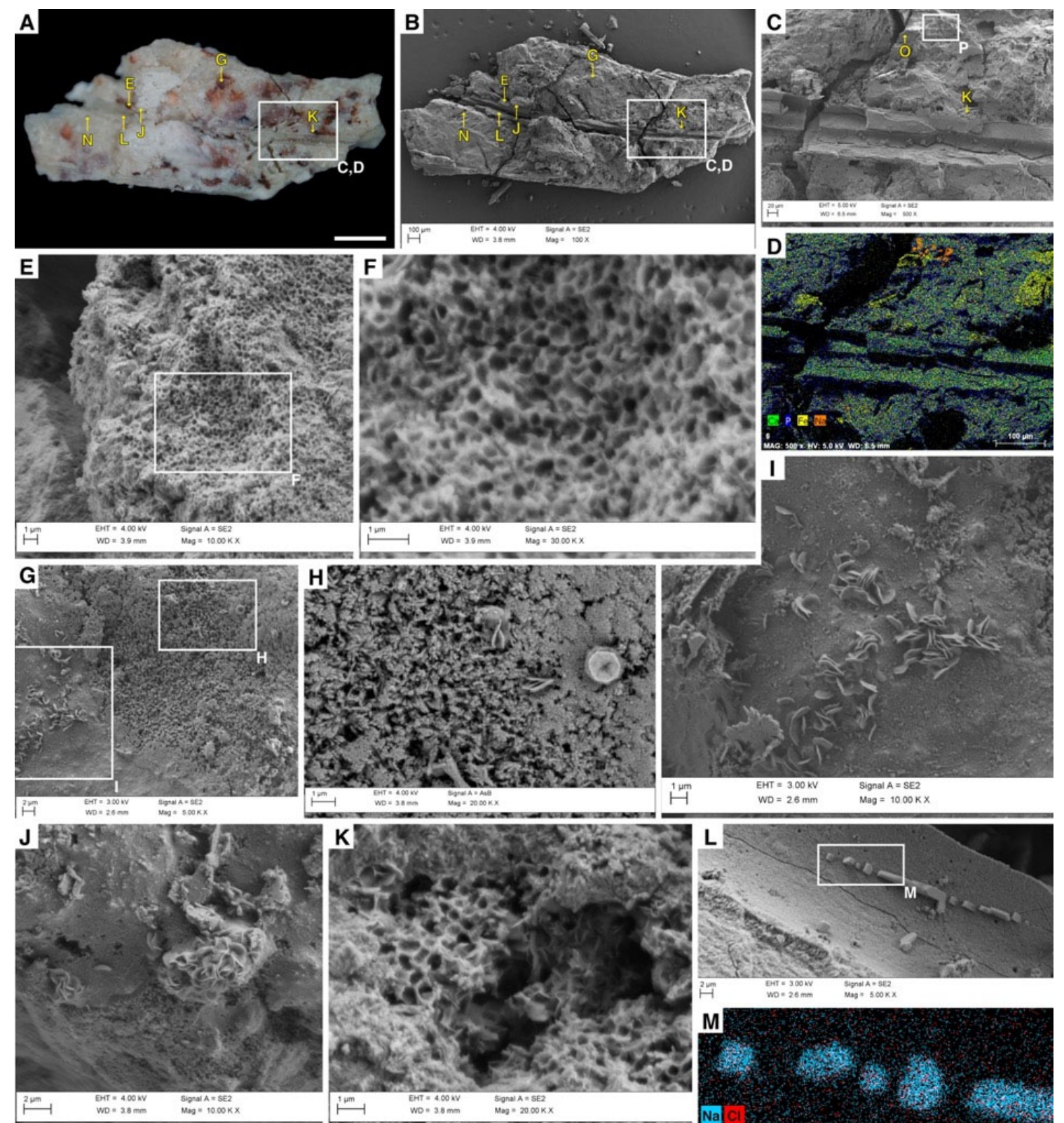

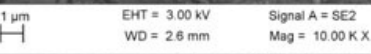
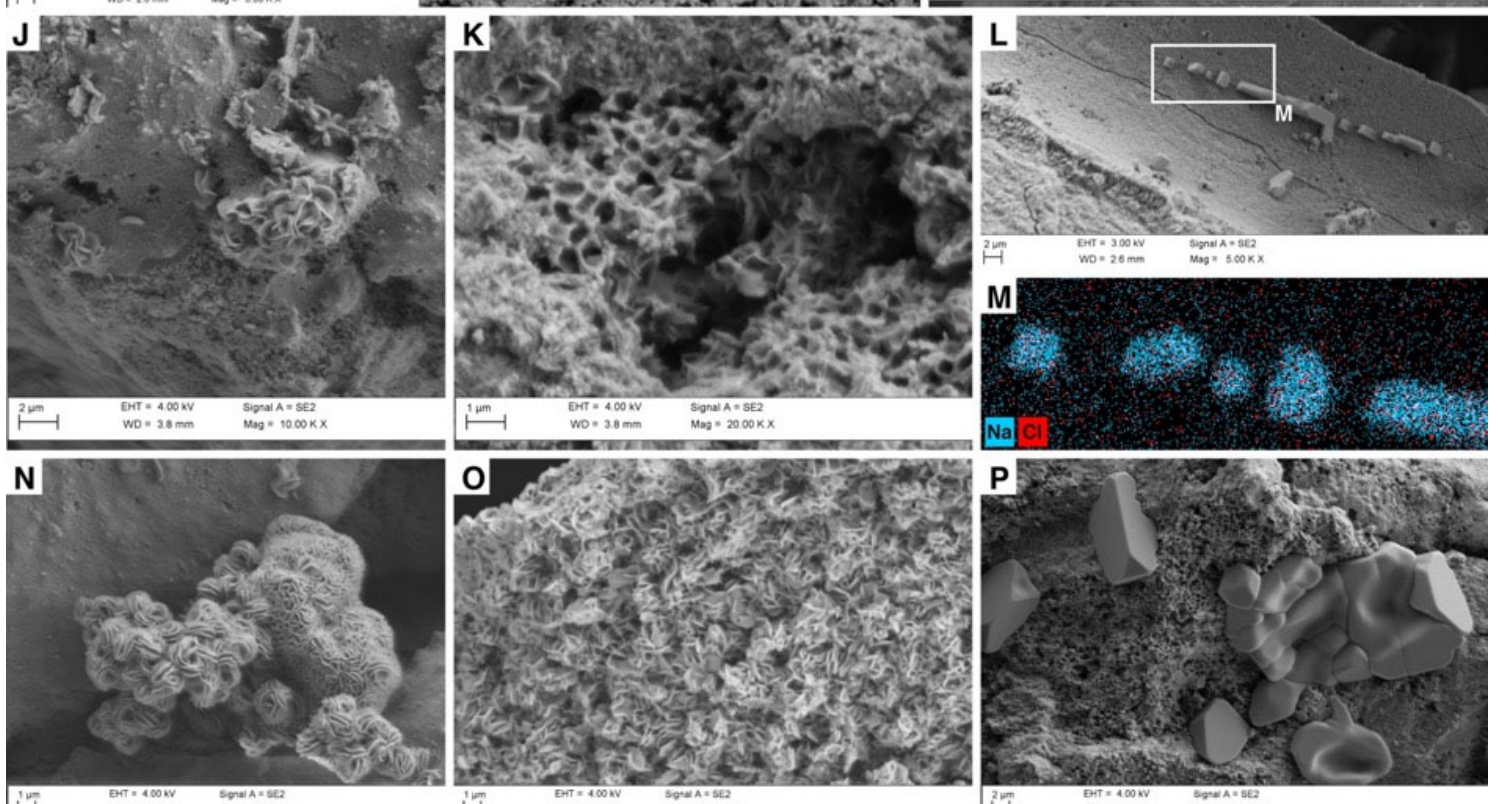

掯

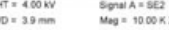

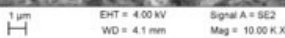

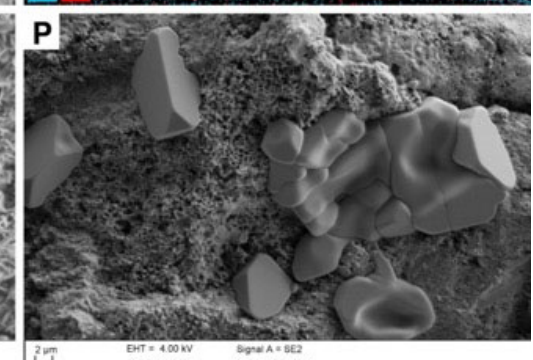

Figure DR2. FEG-SEM imaging and EDX elemental mapping of the loose sample containing soft tissues and bone shown in Fig. 3C from the teleost fish MHNM-KK-OT 10. A, B: Optical photograph (A) and SE image (B) of the entire sample (scale bars: $500 \mu \mathrm{m}$ ). C-P: SEM 
images $(\mathrm{B}, \mathrm{C}, \mathrm{E}-\mathrm{L}, \mathrm{N}-\mathrm{P})$ and $\mathrm{EDX}$ maps $(\mathrm{D}, \mathrm{M})$ at different magnifications (origin: boxes and arrows identified with respective letters). 

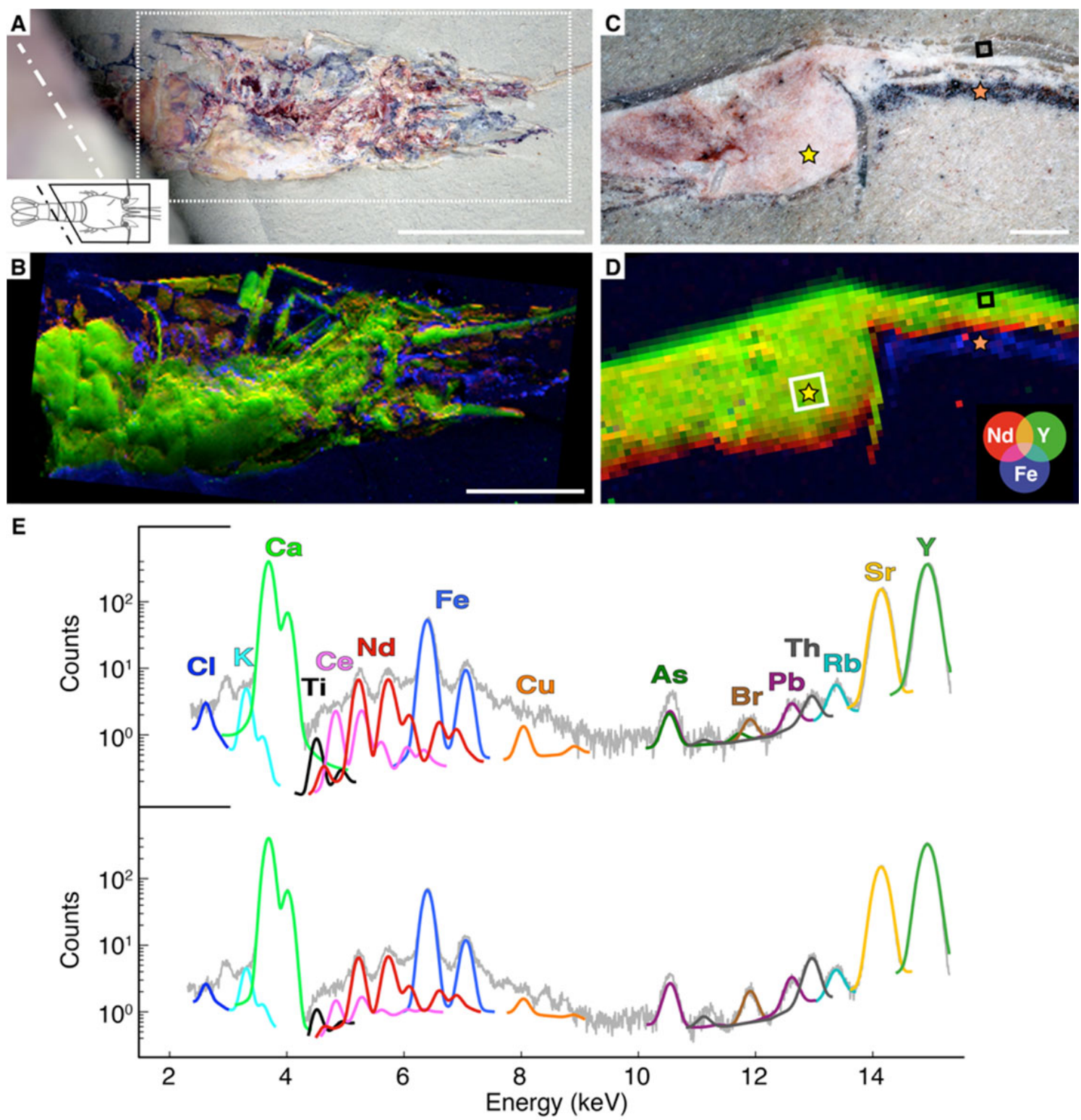

Figure DR3. Synchrotron micro-X-ray fluorescence ( $\mu \mathrm{XRF)}$ mapping of major-to-trace elements of MHNM-KK-OT 01a, and location of XAS and REE pattern data. A: Optical photograph of the penaeid shrimp Cretapenaeus berberus (MHNM-KK-OT 01a). B: False color overlay of neodymium (red), yttrium (green) and iron (blue) distributions (from the area corresponding to the dotted white bow in A). Acquisition parameters: $100 \times 100 \mu \mathrm{m}^{2}$ scan step, 26,751 pixels, $500 \mathrm{~ms}$ counting time per pixel. C: Optical photograph of a cross-section through the fourth somite of the shrimp (MHNM-KK-OT 01c s2f1), prepared following the dotted line in A. D: False color overlay of neodymium (red), yttrium (green) and iron (blue) distributions from the cross-section. Acquisition parameters: $100 \times 100 \mu \mathrm{m}^{2}$ scan step, 13,578 
pixels, $500 \mathrm{~ms}$ counting time per pixel. E: Average XRF spectra and main elemental contributions from the white and black box areas in C-D. Top to bottom spectra are respectively characteristic of the somite soft-tissues (white box, average spectrum from 16 pixels) and cuticle (black box, average spectrum from 25 pixels collected from another, higher resolution map using a 5- $\mu \mathrm{m}$ lateral resolution). Note that the unlabeled peak between $\mathrm{Cl}$ and $\mathrm{K}$ is a XRF Ar-peak due to the excitation of Ar in the air (ca. $0.93 \%$ ) between the sample and the detector. The partial PAAS-normalized REE pattern shown in Fig. DR3A comes from the white box area. The yellow and orange stars indicate the location of XAS spectra collected at the $\mathrm{Ce}_{3}$-edge and Fe K-edge, respectively presented in Fig. DR3B and Figs. 3E, 3F. Scale bars: $A=1 \mathrm{~cm} ; \mathrm{B}=5 \mathrm{~mm} ; \mathrm{C}=1 \mathrm{~mm}$ (this applies also for $\mathrm{D}$ ). 

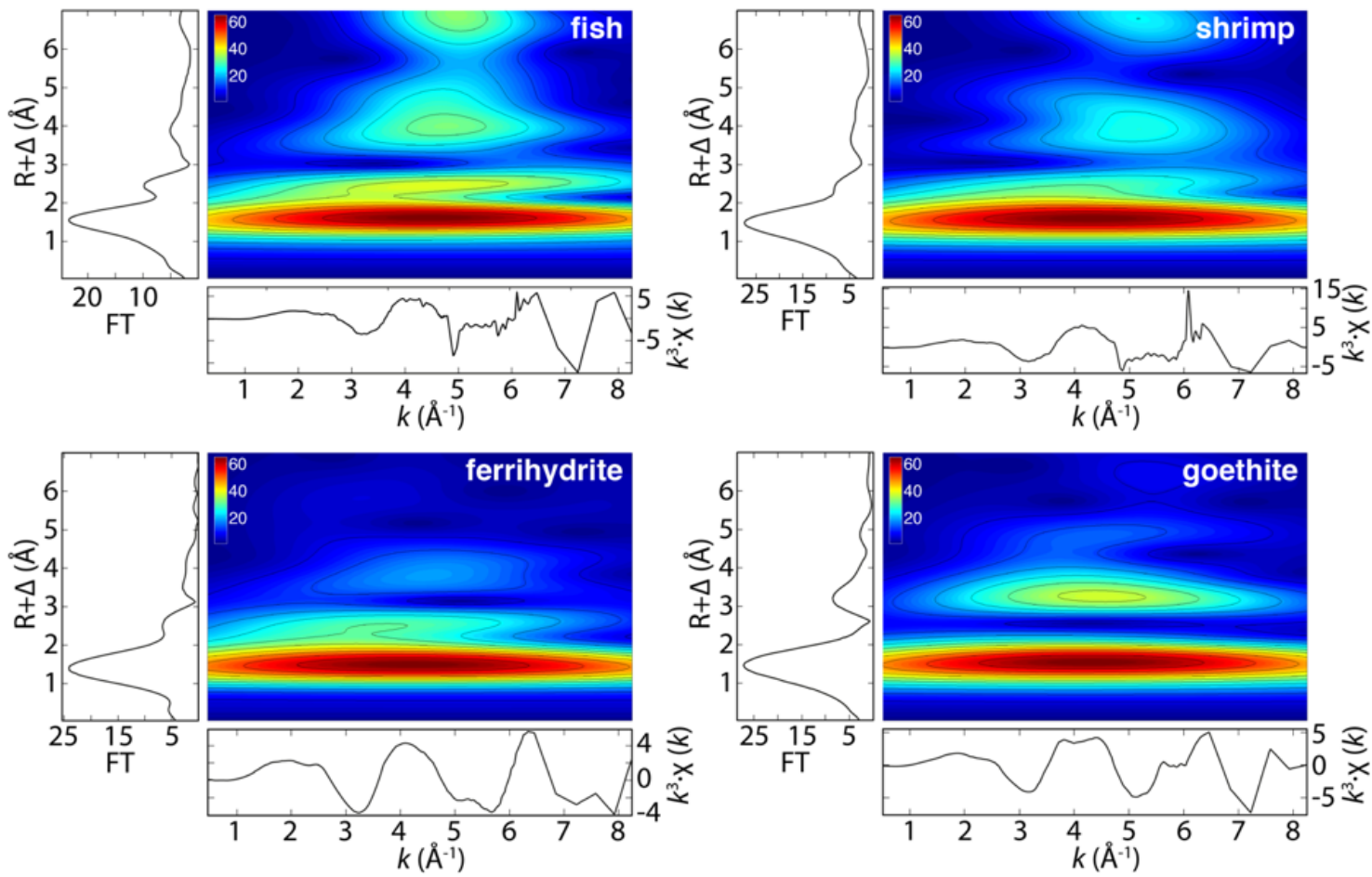

Figure DR4. $\mathrm{k}^{3}$-weighted $\mu$ EXAFS spectra, Fourier transform magnitude and CCWT modulus respectively from the iron-rich grains covering the teleost fish and the shrimp, as well as ferrihydrite and goethite reference materials 

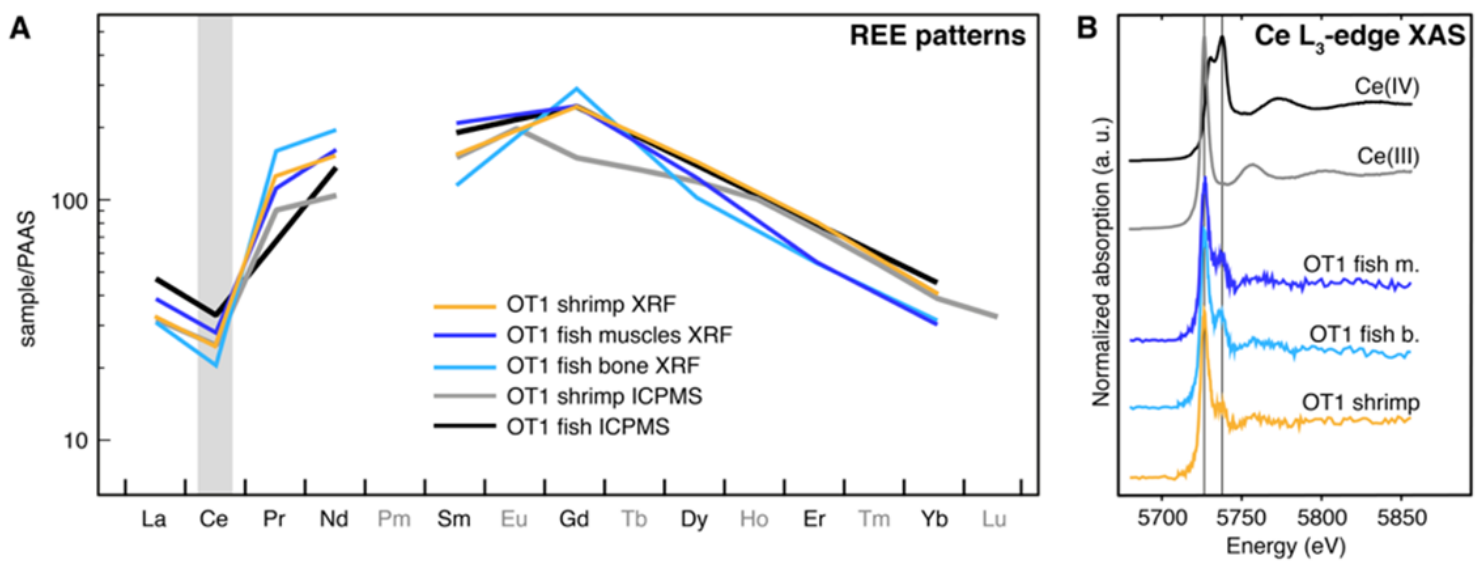

Figure DR5. REE geochemistry. A: Partial PAAS-normalized REE patterns reconstructed from the processed XRF spectra compared to REE patterns based on ICPMS quantifications (the gray square highlights the negative cerium anomaly; elements in gray could not be measured in the partial patterns). B: $\mu \mathrm{XAS}$ spectra at the $\mathrm{Ce} \mathrm{L}_{3}$-edge, compared to $\mathrm{Ce}(\mathrm{III})\left(\mathrm{NO}_{3}\right) 3 \cdot 6 \mathrm{H}_{2} \mathrm{O}$ and $\mathrm{Ce}(\mathrm{IV})\left(\mathrm{SO}_{4}\right)_{2}$ standard spectra (abbreviations: fish $\mathrm{m}$. $=$ fish muscles, fish b. = fish bone; the vertical lines at $5726 \mathrm{eV}$ and $5736 \mathrm{eV}$ highlight the Ce(III) $2 \mathrm{p} \rightarrow\left(4 \mathrm{f}^{1}\right) 5 \mathrm{~d}$ and $\mathrm{Ce}(\mathrm{IV}) 2 \mathrm{p} 3 / 2 \rightarrow\left(4 \mathrm{f}^{0}\right) 5 \mathrm{~d}$ electron transitions, respectively). Spectra are shifted vertically for better visibility. 